\title{
Recent Development in the Molecular Diagnosis of Tuberculosis
}

\author{
Supriya Sarkar
}

\begin{abstract}
Molecular diagnostic tests are the molecular techniques used to detect a nucleic acid of organisms. They have revolutionized the diagnosis of tuberculosis (TB) and drug resistance. Cartridgebased nucleic acid amplification tests (CBNAAT) and line probe assay (LPA) have been accepted in our national tuberculosis control program. CBNAAT has the advantage that it can be done from crude samples and smear negative samples and results can be obtained by 2 hours. But CBNAAT can detect rifampicin resistance only.
\end{abstract}

On the contrary, LPA can detect rifampicin and isoniazid resistance, but the test can only be done at regional or national levels. LPA cannot be done from smear negative or crude specimens. The recent version of LPA (version 2.0) can overcome that problem. Second line LPA can detect the drug resistance against fluoroquinolones, second line injectable drugs and ethambutol (in version 1.0 only). GeneXpert Omni is a portable technique having 4-hour lasting battery using the same cartridge. GeneXpert Ultra has more sensitivity due to larger chamber size and due to two additional probes. Ultra may replace Xpert soon as it will be more effective against smear-negative TB, TB in HIV positive persons, childhood TB, and extrapulmonary TB. With the introduction of molecular diagnostic tests for TB, we will be able to reduce the time of diagnosis of TB and drug resistance. Thereby, we will be able to start first line or second line anti-tubercular chemotherapy earlier, and that will help us to achieve our goal in controlling TB.

Keywords: Cartridge-based nucleic acid amplification tests (CBNAAT), Line probe assay (LPA), Tuberculosis molecular diagnosis.

How to cite this article: Sarkar S. Recent Development in the Molecular Diagnosis of Tuberculosis. Bengal Physician Journal 2018;5(2):10-16.

\section{Source of support: Nil}

Conflict of interest: None

\section{INTRODUCTION}

In 1882, in front of a small gathering of scientists, Sir Robert Kock pronounced the discovery of tubercle bacilli. He got Nobel Prize in 1905. Even today, after more than

Professor and Head

Department of Chest Medicine, College of Medicine and Sagore Dutta Hospital, Kolkata, West Bengal, India

Corresponding Author: Supriya Sarkar, Professor and Head, Department of Chest Medicine, College of Medicine and Sagore Dutta Hospital, Kolkata, West Bengal, India, e-mail: drssupriyo@ gmail.com a century of his discovery, the TB remains elusive. We started with clinical diagnosis, and sooner we realized that the symptoms and signs of TB are nonspecific. With the discovery of X-ray by Wilhelm Röntgen, chest X-ray became a useful tool, but no radiological findings are specific for tuberculosis. Sputum microscopy is cheap and to some extent specific test but it is less sensitive. Culture in solid media is the gold standard, but it takes a longer time. Culture in liquid media can reduce the time, but then they take about 2 weeks. High-resolution tomography (HRCT) and other radiological tools can detect the anatomical changes in the lung and other organs but are not diagnostic of TB.

Like any infective conditions, the diagnosis of TB depends on isolation of organisms or their nuclear products. An ideal test for diagnosis of TB should be rapid, low cost, standardized, point of care test (POCT), automated (requiring minimal training), requiring minimum biosafety measures, and with satisfactory sensitivity and specificity and realistic positive and negative prediction values. POCT is defined as medical diagnostic testing performed outside the clinical laboratory close to patient care centers. Molecular diagnostic tests fulfill many of those criteria.

Molecular diagnostic tests are the molecular techniques used to detect a nucleic acid of organisms, usually after multiplication. They are rapid, and reports are available within hours. They can identify mycobacterium tuberculosis (MTB) complex from cultured isolates or directly from a clinical specimen. They can also detect drug-resistant mutants. There are many types of nucleic acid amplification test (NAAT). They include polymerase chain reaction (PCR), transcription mediated amplification (TMA), line probe assay (LPA), strand displacement amplification (SDA), nucleic acid sequence based amplification (NASBA), reverse transcriptase (RT)-PCR, CBNAAT cartridge based NAAT), etc.

The MTBDRplus (LPA) and Xpert MTB/RIF (CBNAAT) have been endorsed by the World Health Organization (WHO) for the rapid diagnosis of tuberculosis and drugresistant tuberculosis. Revised National Tuberculosis Control Programme (RNTCP) of India has incorporated CBNAAT for use in district and subdistrict levels and LPA for use in national and regional levels. This chapter will mainly concentrate on CBNAAT and LPA. 


\section{Polymerase Chain Reaction}

Kary Mullis in 1993 developed PCR. In PCR, the biological sample is heated to denature double-stranded deoxyribonucleic acid (DNA) into single-stranded DNA (Fig. 1). After cooling, a specific primer is added to produce a template-primer complex. Then nucleotides and heat stable DNA (Taq) polymerase are added to produce first filial generation. First filial generation serves as a template for further DNA synthesis, and the process is repeated to produce millions of copies (exponential amplification after 35 multiplications) around 34 billion copies are produced. Then amplified products are detected by southern blotting or fluorescent/radiolabelled probe hybridization.

The sensitivity and specificity of PCR in TB are 95 and $98 \%$, respectively in smear positive for acid-fast bacilli (AFB) respiratory specimens, and they are 75 to $88 \%$ and $95 \%$ respectively in smear negative culture positive for MTB respiratory specimens. ${ }^{1}$ PCR is more sensitive than a smear but less sensitive than culture for diagnosis of TB. PCR may be positive in dead and dormant bacilli, so it does not always indicate active TB. Also, contamination and laboratory errors can cause false positive results.

On the contrary, a negative PCR result is not sufficient to exclude TB. It has been observed that PCR positivity may vary from sample to sample. PCR is less accurate if the patient had ATD before. The presence of inhibitors like haemin, globulin, beetle nut, etc. can cause false negative results. PCR has the problem of high cost, and it is not a POCT.

\section{Xpert MTB/RIF Assay (CBNAAT/GeneXpert)}

The GeneXpert has been developed by the Foundation for Innovative New Diagnostics (FIND). The Xpert MTB/ RIF assay has been approved by WHO in 2010 and US FDA in 2013 for the diagnosis of tuberculosis in patients getting less than 7 days of therapy. It is a fully automated and quick test (results obtained by 2 hours) that requires minimum bio-safety and training. CBNAAT can also detect rifampicin (RIF) resistance. Dr Mario Ravihlione, director of WHO stop TB department has rightly said, "This new test represents a major milestone for global TB diagnosis and care. It also represents new hope for the millions of people who are at the highest risk of TB and drug-resistant disease."

The GeneXpert MTB/RIF is an automated real-time PCR assay designed for the rapid and simultaneous detection of MTB and 'RIF' resistance within 2 hours. The test utilizes single-use plastic cartridges with multiple chambers that are preloaded with liquid buffers and lyophilized reagent beads. Clinical samples are treated with sodium hydroxide and isopropanol-containing reagent at 3:1 ratio for sputum pellets and at 2:1 ratio for unprocessed sputum samples. Then, they are incubated at room temperature for 15 minutes. The treated sample is then manually transferred to the cartridge, and then that is loaded into the gene Xpert instrument. Subsequent processing is fully automated. Mycobacterium tuberculosis is detected by the five overlapping molecular probes (probes A-E) that collectively are complementary to the entire $81 \mathrm{bp}$ rpo core region. MTB is identified when at least two of the five probes give positive signals with a cycle threshold (CT) of $d^{\prime \prime} 38$ cycles. CT is the number of multiplication of DNA that is required for the detection of DNA of MTB. The basis for detection of RIF resistance is the difference between the first (early CT) and the last (late CT) M. tuberculosis-specific beacon ( $\mathrm{ACT}$ ).

In a large multicentric study including 1730 patients in Peru, Azerbaijan, South Africa, and India with suspected tuberculosis, the test correctly identified $98 \%$ smear-positive tuberculosis and $72 \%$ smear-negative/ culture-positive tuberculosis. ${ }^{2}$ Monoresistance to RIF is rare and at least $90 \%$ of all RIF-resistant clinical isolates are also resistant to INH. Hence, a positive result for RIF resistance is used as a strong surrogate of multi-drug resistant (MDR)-TB. ${ }^{3}$ RIF resistance outside rpo gene is rare and it has been shown that up to 95 to $98 \%$ RIF resistance is caused by mutations in the rpo $\beta$ gene.

Polymerase chain reaction

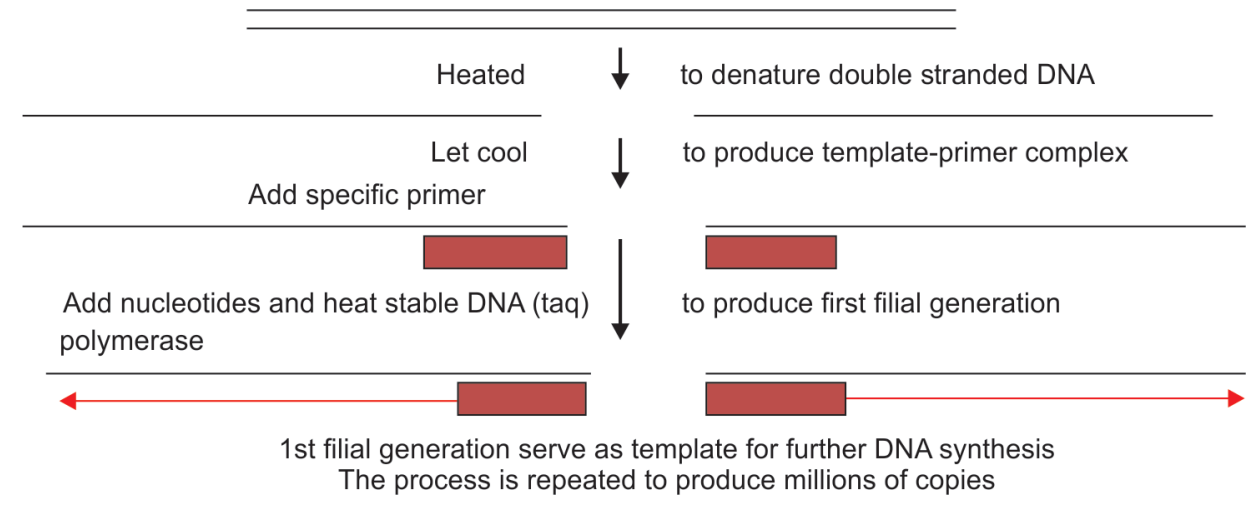

Fig. 1: Steps of PCR 
A meta-analysis (8 studies involving 4461 samples) was carried out comparing gene Xpert with culturepositive TB and TB diagnosed by composite reference standard (CRS). The CRS included a NAAT (other than Xpert), histology, smear, biochemical testing results, presenting signs and symptoms or response to treatment with anti-TB therapy in addition to culture. Xpert sensitivity differed substantially between sample types. In lymph node tissues or aspirates, Xpert pooled sensitivity was $83.1 \%$ ( $95 \%$ CI 71.4 to $90.7 \%$ ) versus culture and $81.2 \%$ (95\% CI 72.4 to $87.7 \%$ ) versus CRS. In cerebrospinal fluid, Xpert pooled sensitivity was $80.5 \%$ (95\% CI 59.0 to $92.2 \%$ ) against culture and $62.8 \%$ (95\% CI 47.7 to $75.8 \%$ ) against CRS. In pleural fluid, pooled sensitivity was $46.4 \%$ (95\% CI 26.3 to $67.8 \%$ ) against culture and $21.4 \%$ (95\% CI 8.8 to $33.9 \%$ ) against CRS. Xpert pooled specificity was consistently $98.7 \%$ against CRS across different sample types. ${ }^{4}$

One study done in pediatric patients showed that in pulmonary samples, CBNAAT detected MTB in $21.4 \%$ sputum/induced sputum samples and in 33\% gastric lavage/aspirate samples. For extrapulmonary samples, the detection rates were $12.7 \%$ for CSF samples, $10 \%$ for pleural fluid samples and $66.7 \%$ for lymph node aspirate samples. They found that in pediatric population sensitivity, specificity, positive predictive value and negative predictive value of CBNAAT in reference to culture were $92.7 \%, 98.9 \%, 97.1 \%$, and $97.2 \%$ respectively, and those were $100 \%, 90.68 \%, 71.42 \%$, and $100 \%$ respectively in reference to Ziehl-Neelsen (ZN) smear. ${ }^{5}$

Our study in tuberculous pleural effusion taking 105 cases (95 TB pleural effusion and 10 TB empyema), showed that pleural fluid acid-fast bacilli smear, mycobacterial culture, and CBNAAT positivity were $8.57 \%, 20 \%$, and $15.23 \%$ respectively. For tuberculous pleural effusion, the sensitivity of CBNAAT (taking culture positivity as reference standard) was 4.76\% [95\% confidence interval (CI) 0.99 to 13.29] and specificity was $87.5 \%$ (95\% CI 71.01 to 96.49$)$. In tuberculous empyema, the sensitivity and specificity were $100 \%$ (95\% CI 66.37 to 100 ) and 100\% (95\% CI 2.5 to 100), respectively. ${ }^{6}$ As bacilli are less in TB effusion than TB empyema, we concluded that the sensitivity of CBNAAT depends on the number of bacilli.

\section{Line Probe Assay/MTBDRplus ("Hain test")}

Line probe assay was introduced by WHO for low resource countries in 2008, that can rapidly screen patients within 2 days. It can detect MTB complex as well as drug-resistant mutants. Demonstration project conducted at Ahmedabad, Hyderabad and Jaipur showed that performance characteristics of LPA were: sensitivity 96\% (CI: 90 to 98\%), specificity 99\% (CI: 95 to $99 \%$ ), positive predictive value $99 \%$ (CI: 95 to $99 \%$ ), and negative predictive value $95 \%$ (CI: 89 to $98 \%$ ). ${ }^{7}$ LPA proved highly accurate in the rapid detection of RIF and isoniazid (INH) resistance. The sensitivity and specificity of LPA were found to be $96 \%$ and $99 \%$ respectively for RIF resistance, and $72 \%$ and $97 \%$ respectively for INH resistance. It has $97 \%$ concordance with LJ medium drug susceptibility testing (DST) for RIF resistance and 79\% for INH resistance.

In LPA, DNA is extracted from MTB complex culture isolates or clinical smear positive samples. Then, speciesspecific regions and resistant-determining regions of DNA are amplified using biotinylated primers. Then, labelled PCR products are hybridized with specific oligonucleotide probes and immobilized on a strip. Then, captured labeled hybrids (Biotin Avidin Alkaline phosphatase system) are detected by colorimetry and that lead to the development of color-bands those can be observed by naked eye.

First line LPA can detect MTB, and resistance to INH and RIF. Drug resistance is detected by the absence of colored band formation with wild-type probe and by the presence of color band formation with commonly occurring specific mutant-type probes (Fig. 2). In that figure, the first strip shows normal MTB. The middle strip shows the bacillus is resistant to RIF and INH (absence of band formation with wild rpo and wild Kat G gene, and presence of band formation with mutant rpo gene and mutant $k a t G$ gene). The last strip shows the bacillus is resistant to INH only (absence of binding with wild inh $A$ gene and presence of binding with mutant inh $A$ gene).

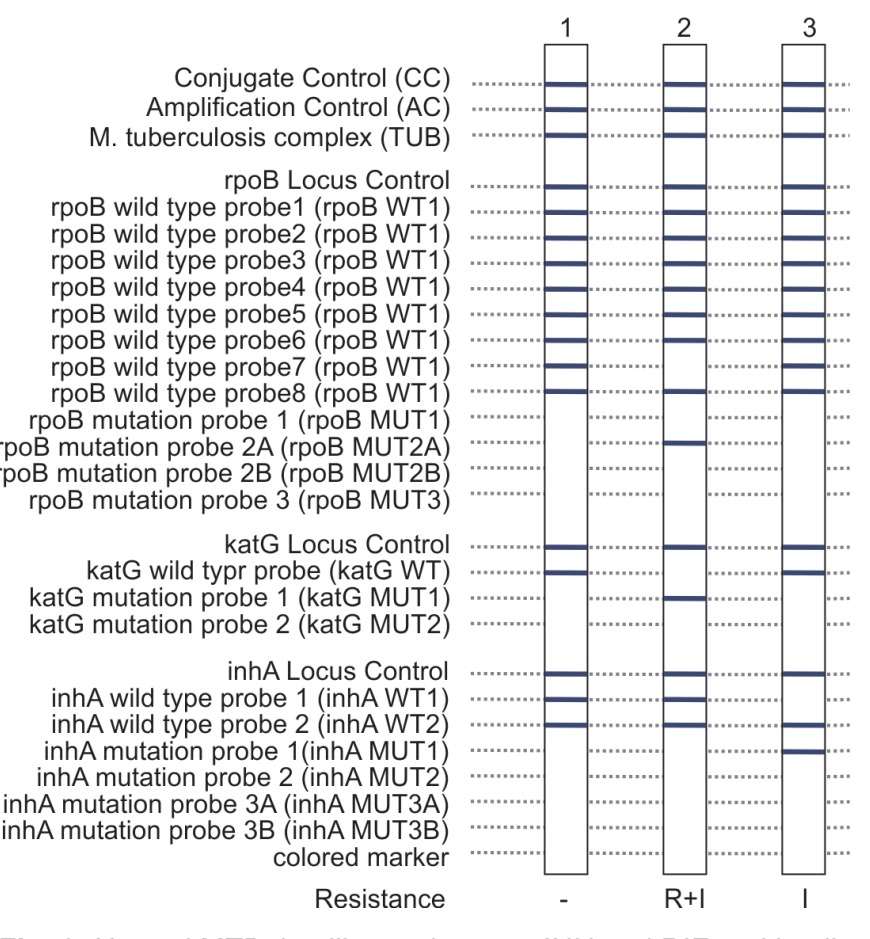

Fig. 2: Normal MTB, bacillus resistant to INH and RIF and bacillus resistant to INH only 
LPA can give some additional information over detection of INH and RIF resistance (MDR-TB). LPA gives information about the sensitivity of high dose INH and ethionamide. Presence of $k a t G$ mutation means high-level INH resistance where high dose INH will not work, but ethionamide will work. On the contrary, the presence of inhA mutation means low-level INH resistance where high dose INH will work, but ethionamide will not work. Both high dose INH and ethionamide will not work if bacilli show both Kat $\mathrm{G}$ and inhA mutations then

\section{LPA vs. CBNAAT}

The major differences CBNAAT and LPA (version 1.0) have been depicted in Table 1. The major problem with LPA is that it can only be applied in smear-positive specimens or culture isolates and it cannot be applied in smear-negative samples and crude samples. That problem has been solved in version 2.0 LPA, where smear negative and crude samples may be tested.

One remarkable study was conducted among 405 suspected drug-resistant tuberculosis. The study compared the efficacy of LPA and Xpert MTB/RIF. ${ }^{8}$ All smearpositive samples $(\mathrm{n}=285)$ were subjected to LPA. LPA results came as, (i) 72 (25.8\%) samples were multidrug resistant, (ii) 62 (22.2\%) showed RIF mono-resistance, (iii) 29 (10.3\%) showed INH mono-resistance, (iv) 116 (41.5\%) were pan-sensitive and (v)6 (2.1\%) samples gave invalid

Table 1: Differences between CBNAAT and LPA

\begin{tabular}{|c|c|c|}
\hline & CBNAAT & LPA (Version 1.0) \\
\hline Automated & $\begin{array}{l}\text { More automated (less } \\
\text { training and bio-safety } \\
\text { required) }\end{array}$ & $\begin{array}{l}\text { Less automated } \\
\text { (more training and } \\
\text { bio-safety measures } \\
\text { are required) }\end{array}$ \\
\hline Technique & $\begin{array}{l}\text { Technically more } \\
\text { robust. }\end{array}$ & $\begin{array}{l}\text { Technically less } \\
\text { robust. }\end{array}$ \\
\hline $\begin{array}{l}\text { Biological } \\
\text { material }\end{array}$ & $\begin{array}{l}\text { Can be done on both } \\
\text { smear positive and } \\
\text { negative cases. }\end{array}$ & $\begin{array}{l}\text { Can be done only } \\
\text { on smear positive } \\
\text { samples. }\end{array}$ \\
\hline $\begin{array}{l}\text { Applicability } \\
\text { with crude } \\
\text { samples }\end{array}$ & $\begin{array}{l}\text { Can be done on crude } \\
\text { clinical specimens. }\end{array}$ & $\begin{array}{l}\text { Difficult to apply } \\
\text { on crude clinical } \\
\text { specimens. }\end{array}$ \\
\hline $\begin{array}{l}\text { Applicability } \\
\text { in persons } \\
\text { living with HIV } \\
\text { infection }\end{array}$ & $\begin{array}{l}\text { More value in person } \\
\text { living with HIV infection }\end{array}$ & $\begin{array}{l}\text { Less value in } \\
\text { persons living with } \\
\text { HIV infection }\end{array}$ \\
\hline $\begin{array}{l}\text { Applicability in } \\
\text { extrapulmonary } \\
\text { TB patients }\end{array}$ & $\begin{array}{l}\text { More value in } \\
\text { extrapulmonary TB } \\
\text { patients. }\end{array}$ & $\begin{array}{l}\text { Less value in } \\
\text { extrapulmonary TB } \\
\text { patients. }\end{array}$ \\
\hline Time taken & $\begin{array}{l}\text { Results obtained within } \\
2 \text { hours }\end{array}$ & $\begin{array}{l}\text { Results obtained in } \\
2 \text { days. }\end{array}$ \\
\hline $\begin{array}{l}\text { Resistance } \\
\text { detected }\end{array}$ & $\begin{array}{l}\text { Can detect only } \\
\text { Rifampicin resistance. }\end{array}$ & $\begin{array}{l}\text { Can detect both } \\
\text { Rifampicin and } \\
\text { Isoniazid resistance }\end{array}$ \\
\hline $\begin{array}{l}\text { Place of } \\
\text { installation }\end{array}$ & $\begin{array}{l}\text { Can be used in district } \\
\text { and subdistrict level. }\end{array}$ & $\begin{array}{l}\text { Can only be used } \\
\text { in national and } \\
\text { regional level. }\end{array}$ \\
\hline
\end{tabular}

results. Sixty-two RIF mono-resistant samples were subjected to Xpert MTB/RIF using cartridge version G4. Three (4.8\%) samples gave an error, $38(61.4 \%)$ showed RIF resistance, while 21 (33.8\%) were susceptible to RIF. Among pan-susceptible samples, 83 samples were tested for Xpert MTB/RIF. Results showed that $5.1 \%$ were RIF resistant. The 25 discrepant samples were further subjected to liquid culture by mycobacterial growth indicator tube (MGIT-960) and DST. The MGIT-960 results showed $100 \%$ agreement with LPA results but only $64.4 \%$ agreement with Xpert MTB/RIF results.

\section{Reliability of Xpert MTB/RIF}

One recent study questioned the reliability of RIF resistance report of Xpert MTB/RIF in samples with very low detection of MTB. ${ }^{9}$ The study was designed to check the performance of Gene Xpert in those clinical samples where MTB detected by GeneXpert were very low. Out of 2024 specimens tested positive with gene Xpert, 410 specimens were MTB detection level "very low." The number of RIF resistance was 35 among 'MTB detected very low' specimens, and those samples were put in culture. Only 22 produced positive cultures, and those were subjected to confirmatory testing for RIF resistance like DST, rpo sequencing and spoligotyping. Surprisingly, 12 out of 22 GeneXpert "RIF resistant" isolates showed RIF sensitivity on DST. By rpo $\beta$ sequencing those isolates it was proved that those samples did not harbor rpo mutations and those were actually RIF sensitive. Remaining 10 out of 22 samples were truly RIF resistant. In samples with very low bacillary load, the false RIF resistance detection by Xpert MTB/RIF was 54.5\%. The observed false-resistant results were confirmed with three different gene Xpert cartridges. Though not recommended, it is suggested that the detection of RIF resistance by Xpert MTB/RIF in very low detection samples should be subjected to LPA or culture-based DST. This may be true in smear-negative samples, pediatric samples, samples in HIV positive persons and extrapulmonary TB.

The negative predictive value of Xpert MTB/RIF assay in detecting RIF resistance is over $99 \%$ in settings with both low and high prevalence of RIF resistance, i.e., a negative result accurately excludes the possibility for RIF resistance. The positive predictive value (PPV) for RIF resistance using Xpert MTB/RIF depends on the underlying prevalence of RIF resistance. In settings or in patient groups where RIF resistance is rare, the PPV of Xpert MTB/RIF testing is adversely affected.

\section{Time Factor}

On average, AFB smear microscopy takes one I day, liquid culture takes 17 days, solid culture takes more than 30 
days, Xpert MTB/RIF takes 2 hours, and LPA takes 2 days. It has been found that the average time to start treatment in smear-negative TB before the availability of Xpert MTB/RIF was 58 days, while that can be brought down to 4 days with the availability of Xpert MTB/RIF. The detection of RIF resistance (MDR TB) was on average 75 days for phenotypic DST, while that can be brought down to 1 day with Xpert MTB/RIF.

\section{Second Line LPA (MTBDRsI)}

The second line LPA (LPAsl) is a DNA-based test that identifies genetic mutations for resistant to fluoroquinolones (FQs) and second line injectable drugs (SLIDs). WHO recommended LPAsl as an initial test for detection of additional resistance to FQ and SLID in patients with confirmed RIF-resistant TB or MDR-TB. These recommendations apply to the use of LPAsl for the direct testing of sputum specimens and indirect testing on culture isolates in adults as well as in children. For SLID, the results highly correlate with culture-based phenotypic resistance. For FQ, the results correlate better with ofloxacin/levofloxacin resistance than moxifloxacin resistance.

MTBDRsl version 2.0 is now available. The main advantage of version 2.0 is that the test can be done from smear negative and crude biological samples. The only disadvantage is that ethambutol sensitivity cannot be detected in version 2.0. The difference between version 1.0 and 2.0 has been depicted in Table 2.

The gene mutation of different regions has remarkable clinical significance. For FQs, gyrA gene mutation (1 and 2) signifies low-level resistance to FQs and moxifloxacin

Table 2: Difference between version 1.0 and version 2.0 of MTBDRsI

\begin{tabular}{|c|c|c|}
\hline $\begin{array}{l}\text { MTBDRsl } \\
\text { versions }\end{array}$ & Version 1.0 & Version 2.0 \\
\hline Samples & $\begin{array}{l}\text { Smear-positive } \\
\text { specimens and } \\
\text { culture isolates }\end{array}$ & $\begin{array}{l}\text { Smear-positive and } \\
\text { smear-negative } \\
\text { specimens and culture } \\
\text { isolates }\end{array}$ \\
\hline Detection & $\begin{array}{l}\text { M. tuberculosis } \\
\text { complex and } \\
\text { resistance to } \\
\text { fluoroquinolones, } \\
\text { SLIDs and } \\
\text { ethambutol }\end{array}$ & $\begin{array}{l}\text { M. tuberculosis } \\
\text { complex and } \\
\text { resistance to } \\
\text { fluoroquinolones and } \\
\text { SLID }\end{array}$ \\
\hline $\begin{array}{l}\text { Fluoroquinolone } \\
\text { resistance }\end{array}$ & $\begin{array}{l}\text { Mutations in } \\
\text { resistance- } \\
\text { determining region } \\
\text { of the gyrA gene }\end{array}$ & $\begin{array}{l}\text { Mutations in } \\
\text { resistance-determining } \\
\text { regions of the gyrA } \\
\text { and gyrB genes }\end{array}$ \\
\hline SLID resistance & $\begin{array}{l}\text { Mutations in } \\
\text { resistance } \\
\text { determining region } \\
\text { of the rrs gene }\end{array}$ & $\begin{array}{l}\text { Mutations in } \\
\text { resistance determining } \\
\text { region rrs gene and } \\
\text { the eis promoter } \\
\text { region }\end{array}$ \\
\hline $\begin{array}{l}\text { Ethambutol } \\
\text { resistance }\end{array}$ & $\begin{array}{l}\text { Mutations in the } \\
\text { embB gene }\end{array}$ & Not included \\
\hline
\end{tabular}

in high dose can be given, whereas, a mutation at $3 \mathrm{~A}$ to $\mathrm{D}$ regions signifies high-level FQ resistance and high dose moxifloxacin will not be effective. For SLIDs, rrs gene mutation signifies a high level of resistance against amikacin (AMK), kanamycin ( KAN) and capreomycin (CAP); whereas, mutation at 'eis promoter region' signifies KAN will not work but AMK, CAP can be given.

\section{Gene Xpert Omni}

A new device called the GeneXpert Omni is currently under development as a point of care test. It will use the same cartridges as gene $X$ pert. The main advantages are that the instrument is smaller and lighter (portable), and less expensive. GeneXpert Omni has a built-in 4-hour battery so that it can work in areas without electricity.

\section{Xpert MTB/RIF Ultra Assay}

Xpert MTB/RIF ultra assay has been launched in 2017. On 24 March 2017 WHO recommended the use of Xpert MTB/RIF ultra in all settings and it will gradually replace the current Xpert MTB assay.

The new Xpert MTB/RIF Ultra cartridge can be used on the same Gene Xpert equipment (Gene Xpert instrument, computer, monitor, keypad, barcode reader) and will be available at the same concessional price of USD 9.98 for all eligible countries. Changes inside the ultra cartridge include a larger chamber (double size) for DNA amplification and two additional TB detecting probes targeting IS6110 and IS1081 gene of MTB. Both are responsible for the increase in sensitivity of the ultra-assay.

Ultra assay is much more sensitive than Xpert. It can detect MTB where the concentration of bacilli is as low as 16 bacilli per $\mathrm{ml}$ compared to 131 per $\mathrm{ml}$ sputum for Gene Xpert). It is likely to be as sensitive as liquid TB culture. The ultra cartridge will be predictably better particularly in specimens with low numbers of bacilli, like smear-negative culture-positive specimens, persons with HIV co-infection, in pediatric specimens and extrapulmonary specimens. Ultra detects RIF-resistance as efficiently as Xpert, but the specificity of ultra is likely to be higher due to improvements in assay design. The accuracy in the detection of RIF resistance was also better although not enough data were available to conclusively confirm this.

The increased sensitivity of the ultra assay is almost exclusively because of its low TB detection limit. The increased sensitivity may be offset by a decrease in specificity. Ultra may also be more prone to detect small numbers of non-replicating or nonviable bacilli, particularly in patients with a recent history of TB treatment, reducing the specificity of the assay. Reduced specificity may give rise to false positive results for TB detection. 
In the FIND-coordinated studies, such results occurred in fewer than $5 \%$ of persons with signs or symptoms suggestive of TB. Because of the internal molecular configuration of the Ultra cartridge, RIF resistance detection is not similarly affected.

\section{CONCLUSION}

It is claimed that Xpert MTB/RIF ultra will be a game changer for overcoming the challenge of fast diagnosis of TB in smear-negative patients, and we are moving a step closer towards TB elimination. But all tests, however effective they are, bring some problems with them. For example, increased sensitivity is associated with decreased specificity.

"We should not treat laboratory results but we should treat the patient." That dictum is universally true. We have registered many successes in laboratories, but we failed miserably in the field conditions to apply that knowledge. As a result, we cannot achieve our goal in controlling tuberculosis.

Even after applying modern diagnostic technology, a substantial number of tuberculosis cases cannot be confirmed microbiologically. "Microbiologically confirmed tuberculosis" means confirmation of the diagnosis of TB by isolating TB bacilli or their nuclear product from biological samples. On the other hand, "clinically diagnosed tuberculosis" means the cases diagnosed by clinical, radiological, biochemical and other methods those are suggestive of TB. With the help of modern molecular technology, our aims should be to increase the number of microbiologically confirmed TB and to reduce the number of clinically diagnosed TB. Molecular diagnostic tests cannot completely replace previous diagnostic tests, though they have the additional advantage of quick detection of TB and drug resistance. Molecular diagnostic tests, like that of other diagnostic tests, should be utilized judiciously and obviously, they take us closer to TB control.

\section{REFERENCES}

1. Laraque F, Griggs A, Slopen M, Munsiff SS. Performance of nucleic acid amplification tests for diagnosis of tuberculosis in a large urban setting. Clin Infect Dis 2009;49:46

2. Boehme CC, Nabeta P, Hillemann D, et al. Rapid molecular detection of tuberculosis and rifampin resistance. N Engl J Med 2010;363:1005..

3. Van Rie A, Page-Shipp L, Scott L, Sanne I, Stevens W. Xpert MTB/RIF for point of care diagnosis of TB in high-HIV burden, resource-limited countries: hype or hope? Expert Rev Mol Diagn 2010;10:937-946.

4. Claudia M. Denkinger, Samuel G. Schumacher, Catharina C. Boehme, Nandini Dendukuri, Madhukar Pai and Karen R. Steingart. Xpert MTB/RIF assay for the diagnosis of extrapulmonary tuberculosis: a systematic review and meta-analysis. Eur Respir J 2014; in press.

5. Anshu K, Das S, Paul DK. A Study on the Role of Cartridge Based Nucleic Acid Amplification Test (CBNAAT) for Diagnosing Pediatric Tuberculosis in a Tertiary Care Hospital in Eastern India. Acad J Ped Neonatol 2018;6(3):555745.

6. Biswas D, Mukherjee S, Begum S, Paul A, Ghosh P, Sarkar S. Role of Cartridge Based Nucleic Acid Amplification Test in Diagnosis of Tuberculous Pleural Effusion Compared to Tuberculous Empyema in HIV Seronegative Patients. Int J Sci Stud 2017;5(6):125-129.

7. Raizada N, Sachdeva KS, Chauhan DS, Malhotra B, Reddy $\mathrm{K}$, Dave PV, et al. A multi-site validation in India of the line probe assay for the rapid diagnosis of multi-drug resistant tuberculosis directly from sputum specimens. PLoS One. 2014 Feb 19;9(2):e88626.

8. Rufai SB, Kumar P, Singh A, Prajapati S, Balooni V, Singh S. Comparison of Xpert MTB/RIF with Line Probe Assay for Detection of Rifampin-Monoresistant Mycobacterium tuberculosis. Journal of Clinical Microbiology [Internet]. American Society for Microbiology; 2014 Mar 19;52(6):18461852.

9. Ocheretina O, Byrt E, Mabou M-M, Royal-Mardi G, Merveille $\mathrm{Y}-\mathrm{M}$, Rouzier V, et al. False-positive rifampin resistant results with Xpert MTB/RIF version 4 assay in clinical samples with a low bacterial load. Diagnostic Microbiology and Infectious Disease [Internet]. Elsevier BV; 2016 May;85(1):5355 . 
